\title{
A Nonintrusive Load Identification Method for Residential Applications based on Quadratic Programming
}

\author{
Shunfu Lin ${ }^{\text {a }}$, Lunjia Zhao ${ }^{\text {a }}$, Fangxing $\mathrm{Li}^{\mathrm{b}}$, Qingqiang Liu ${ }^{\mathrm{a}}$, Dongdong $\mathrm{Li}^{\text {a }}$, Yang Fu ${ }^{\mathrm{a}, \mathrm{c}}$ \\ ${ }^{a}$ : Dept. of EE, The Shanghai University of Electric Power; Shanghai, 200090; China. \\ ${ }^{\mathrm{b}}$ : Dept. of EECS, The University of Tennessee; Knoxville, TN, 37996; USA. \\ c: Contact author: mfudong@126.com, +86-133-7189-6889.
}

Abstract-The decomposition of household power consumption is highly desired for scheduling household appliances and reducing home energy consumption. This paper presents a novel nonintrusive load identification method based on quadratic programming. Extensive simulation and laboratory tests have demonstrated that the proposed technique can provide adequate load identification accuracy for residential energy monitoring such that it is suitable for nonintrusive load monitoring (NILM) systems in residential and commercial buildings.

Keywords-Appliance identification, load signature, nonintrusive load monitoring (NILM), quadratic programming.

\section{Introduction}

With the development of smart grid and increasing awareness of energy conservation, the decomposed data at individual load levels in households or buildings is more desired for load scheduling and demand response programs [1]. Past research works indicated that the visualization of home electricity use can reduce $4 \sim 15 \%$ of power consumptions [2]. Two approaches are available to monitor the energy consumption of each appliance in residential dwellings: the traditional approach is to employ separate sensors (i.e., smart plugs) for each load to be monitored. While this may provide accurate measurement of each appliance with a smart plug, this does have the disadvantages of high hardware cost and additional complexity of communication network (e.g., for smart plugs to communicate with each other). In contrast, a newer approach is to employ intelligent algorithms to break down the total power 
consumption, which is called nonintrusive load monitoring (NILM) technique. In general, the traditional approach with separate sensors can be viewed as a high-end solution for accurate measurement, while the new NILM approach can be viewed as a low-cost solution to provide rough energy consumption profiles for customer's awareness of energy consumption and future guideline of appliance scheduling.

NILM was addressed originally at MIT by George W. Hart [3] in the 1980's, and has attracted many interests [4-16] in recent years due to the fast development of smart metering technologies. NILM employs a centralized monitoring device installed at the main breaker level, combined with intelligent load identification technologies to break down the operational information and power consumption of each appliance. NILM can be useful for homeowners and building managers to monitor energy consumption on an appliance-by-appliance basis without having to install dedicated sensors. Large companies (e.g., Intel and Belkin) and small firms (e.g., Onzo in the US and Navetas in the UK) have initiated aggressive research and development efforts in energy monitoring with NILM data [4]. A novel senor embedded with NILM for home energy use monitoring was also developed by a research team at University of Alberta [5].

The original and extended NILM methods by MIT [3] use low-frequency hardware devices that only provide steady, coarse, and macroscopic signatures such as active and reactive power (P-Q). Recently, most researchers have agreed that the high-frequency hardware installation, which is capable of providing not only the conventional steady signatures but the microscopic transient signatures, can reach high accuracy of appliance detection and identification. The microscopic signatures include harmonics, transient power, and geometrical properties of V-I curves, and so on [6]-[10].

Several intelligent algorithms have been proposed for appliance identification by researchers in the past a few years. In [14], the artificial neural network (ANN) was used to 
identify the appliance by simply teaching the ANN to learn specific features. In [15]-[16], the neural network was also used for the electrical appliance identification. The previous works in the literature based on intelligent systems which are based on a large amount of training from operational data. This training effort work will hinder the industrial application of the NILM technologies. There are still no complete available NILM solutions till now such that further research is still needed [4].

This paper presents an intelligent algorithm based on quadratic 0-1 programming for the residential appliance identification. The algorithm can be applied into the monitors or sensors installed at the main breaker of a residential home. A simulation experiment platform in laboratory was built up for testing the proposed algorithms. Ten different types of home appliances are employed in laboratory tests to verify the identification accuracy. The test results validate that the identification accuracy of the proposed algorithm is around $90 \%$.

\section{Residential Load Signatures}

The following signal signatures extracted for the voltage and current signals are employed in this paper.

\subsection{Current waveform signatures}

Three signal signatures can be extracted from a current waveform as follows:

$$
\begin{aligned}
I_{\mathrm{rms}} & =\sqrt{\frac{1}{n} \sum_{k=0}^{n} i(k)^{2}} \\
I_{\mathrm{p}} & =\max (i(k)) \\
I_{\mathrm{CF}} & =\frac{I_{p}}{I_{r m s}}
\end{aligned}
$$

where $I_{\mathrm{rms}}, I_{\mathrm{p}}$ and $I_{\mathrm{CF}}$ are the root mean square value, peak magnitude and crest factor, respectively, of the current waveform.

\subsection{Current harmonic signatures}

The harmonic components can be calculated by the discrete Fourier transform. The 
total harmonic distortion (THD) is written as

$$
T H D_{I}=\sqrt{\sum_{h=2}^{H} I_{h}^{2}} / I_{1}
$$

Typical harmonic orders (e.g., $2^{\text {nd }}, 3^{\text {rd }}$, and $5^{\text {th }}$ ) as well as the THD can be used for appliance identification. The typical order harmonics can be computed with the common discrete Fourier transform (DFT).

\subsection{Active and reactive power signatures}

The active and reactive power are calculated by the following two equations, respectively:

$$
\begin{gathered}
P=\sum_{k=0}^{\infty} P_{k}=\sum_{k=0}^{\infty} U_{k} I_{k} \cos \left(\Phi_{k}\right) \\
Q=\sum_{k=0}^{\infty} Q_{k}=\sum_{k=0}^{\infty} U_{k} I_{k} \sin \left(\Phi_{k}\right)
\end{gathered}
$$

\subsection{Geometrical properties of V-I curves}

Figure 1 shows the $V$ - $I$ curves of four typical appliances such as a fluorescent lamp, an electric heating unit, a refrigerator and a microwave. The central line is the straight line between the maximum and minimum point of the current amplitudes. The slope of the central line is adopted as a signal signature for appliance identification. In addition, the enclosed envelope area of the $V-I$ curve can be also used for identification.

\subsection{Appliance signature database}

A manual registration (MR) process is necessary and suggested to establish the appliance signature database when the monitor is initially installed. For example, if the house owner desires to register a microwave oven, he/she should switch off the other appliances first, and then switch on the microwave oven for a certain duration and then switch it off. The monitor or sensor with synchronous communication function can record the voltage and current signals when the microwave oven is switched on. 
The switching on, switching off, and operation status change of an appliance is called as an Operation Event (OE). An OE detection scheme will be triggered and detect the $\mathrm{OE}$ occurrence counts. A cumulative sum (CUSUM) method is adopted to detect the $\mathrm{OE}$ occurrence, which will be introduced in Section III. Figure 2 shows the current waveform recorded by a monitor or sensor when an OE occurs. At the time of $0.25 \mathrm{~s}$ an appliance is switched on. The difference of the current data between window 4 and window 3 is considered as the working current of switched-on appliance:

Appliance Current=Window 4 Current -Window 3 Current

In this paper, each of window 3 and window 4 contains 4 cycles, while window 1 and window 2 each contains 2 cycles. It can be generally assumed that the appliance operation does not affect the supply voltage. Once the appliance current waveforms are obtained, the signatures will be extracted from the monitoring system and saved into a database.

Following the MR process, the signature database of all appliances connected under the main breaker of a residential household can be established.

In previous sensitivity analyses for transmission contingency, a line outage is typically transformed to changes of bus injections. Different transformations were introduced in the literature [22] [23], as previously mentioned. In this section, a new transformation is proposed with a rigorous mathematical proof, and the outage sensitivity factors based on the transformation are developed.

\section{NILM Load Identification Principle}

\subsection{Load identification scheme}

Without losing generality, we may assume that the calibration signature matrix of the appliances connected under the main breaker can be expressed as follows: 


$$
\Psi=\left[\begin{array}{cccc}
\psi_{11} & \psi_{12} & \cdots & \psi_{1 N} \\
\psi_{21} & \psi_{22} & \cdots & \psi_{2 N} \\
\vdots & \vdots & \ddots & \vdots \\
\psi_{M 1} & \psi_{M 2} & \cdots & \psi_{M N}
\end{array}\right]
$$

where $\psi_{\mathrm{ji}}$ is the $j^{\text {th }}(j=1,2,3, \ldots, M)$ signature value of the $i^{\text {th }}$ appliance $(i=1,2,3, \ldots, N) . N$ is the number of the appliances in the household and $M$ is the number of the selected signatures.

Let the matrix of the operation status of the appliances be

$$
X=\left[\begin{array}{llll}
x_{1} & x_{2} & \cdots & x_{N}
\end{array}\right]^{T}
$$

where $x_{\mathrm{i}} \epsilon\{0,1\}$ is the operation status of the $i^{\text {th }}$ appliance. Here, 0 or 1 represents the appliance status, i.e., switched off for 0 and switched on for 1 .

Assume the operation status matrix $X$ is known. Thus, the ideal signature matrix $Y$ can be expressed by

$$
Y=\left[\begin{array}{llll}
y_{1} & y_{2} & \cdots & y_{M}
\end{array}\right]^{T}=\Psi X
$$

The monitor installed at the main breaker measures the signals of the voltage and current. The measured signature matrix $Y^{\prime}$ calculated from the voltage and current signals is expressed as follows:

$$
Y^{\prime}=\left[\begin{array}{llll}
y_{1}^{\prime} & y_{2}^{\prime} & \cdots & y_{M}^{\prime}
\end{array}\right]^{T}
$$

where $y_{j}^{\prime}(j=1,2,3, \ldots, M)$ is the $j^{\text {th }}$ measured signature value.

Assume that the residual sum of squares between the measured $Y^{\prime}$ and the ideal $Y$ is $\varepsilon$, we have

$$
Y^{\prime}=Y+\varepsilon=\Psi X+\varepsilon
$$

where

$$
\begin{aligned}
& \varepsilon=\left[\begin{array}{llll}
\varepsilon_{1} & \varepsilon_{2} & \cdots & \varepsilon_{M}
\end{array}\right]^{T} \\
& \varepsilon_{j}=\left(y_{j}^{\prime}-y_{j}\right)^{2}=\left(y_{j}^{\prime}-\sum_{i=1}^{N} \psi_{i j} x_{i}\right)^{2}
\end{aligned}
$$

The key problem here is to find the operation status matrix $X$ which lets the residual sum of squares $\varepsilon$ to be the minimum. 
The constraint function is given by:

$$
\sum_{i=1}^{N} x_{i}=1 \quad x_{i}=\{0,1\}
$$

The minimum value of the square of $\varepsilon$ can be written as

$$
\begin{aligned}
& \min \mathrm{E}=\varepsilon^{T} \varepsilon \\
& =\left(Y^{\prime}-\Psi X\right)^{\mathrm{T}}\left(Y^{\prime}-\Psi X\right) \\
& =Y^{\mathrm{T}} Y^{\prime}-2 Y^{\mathrm{T}} \Psi X+\frac{1}{2}\left(X^{T} \Psi^{T} 2 \Psi X\right)
\end{aligned}
$$

It is observed that (15) can be transformed to a quadratic 0-1 programming model which is given by

$$
\left\{\begin{array}{c}
\min E=Y^{\mathrm{T}} Y^{\prime}-2 Y^{\mathrm{T}} \Psi X+\frac{1}{2}\left(X^{T} \Psi^{T} 2 \Psi X\right) \\
\sum_{i=1}^{N} x_{i}=1 \\
x_{i}=\{0,1\}
\end{array}\right.
$$

The above quadratic 0-1 programming function in (16) can be solved easily with the discrete method such as the exhaustive method or implicit enumeration method [17] in the number of appliances in most cases. The detailed solution process will not be introduced here since it is well established in the literature [17].

\subsection{Operation event detection scheme}

It is necessary to detect the operation event to calculate the signature matrix $Y^{\prime}$ for the appliance identification. A sliding window based on the cumulative sum (CUSUM) method [18] is applied here for the OE detection. CUSUM is a sequential analysis technique and typically used for change detection, which is highly suitable for the OE detection in this work.

Define two cumulative sums named as $s_{k}^{+}$and $s_{k}^{-}$as the follows:

$$
\begin{gathered}
s_{k}^{+}=\max \left\{0, s_{k-1}^{+}+x_{k}-(\mu+\beta)\right\} \quad k=1,2,3, \ldots \\
s_{k}^{-}=\min \left\{0, s_{k-1}^{-}+x_{k}-(\mu+\beta)\right\} \quad k=1,2,3, \ldots
\end{gathered}
$$

where $\mu$ is the mean value at the previous time; $\beta$ is the noise signal. 


\subsection{Procedure of the proposed NILM algorithm}

The voltage and current signals are monitored and recorded by a hardware installation called as a monitor at the main breaker. Figure 3 shows the procedure of the proposed NILM algorithm that is embedded inside the monitor.

First, when the monitor is installed initially, a signature database is established through a manual registration process. Once the monitor is started up, it reads the signatures of each appliance from the database to form the matrix $\Psi$. The CUSUM algorithm is recalled to detect the $\mathrm{OE}$ occurrence. Once an $\mathrm{OE}$ occurs, the voltage and current signals are recorded to extract the signal signatures. The function expressed in (17) will be established and solved with Lagrange multiplier method to obtain the matrix $X$ of the operation status of appliances. Thus, it will be identified which appliance is switched on or off from the matrix $X$, and then the electricity consumption and other power parameters can be calculated.

An experimental platform was implemented to verify the feasibility of the proposed algorithm, as shown in Figure 4. The platform includes five different appliances such as a fan (FAN), a desk light (DL), a water fountain (WF), a microwave oven (MO), and an electric heater $(\mathrm{EH})$.

A software tool with a graphic-user interface (GUI) platform based on the Matlab/Simulink was also developed to present the proposed NILM algorithm, as shown in Figure 5. The demonstration platform consists of the main interface, parameter-setting interface, signature database interface, electricity visualization interface and historical data query interface. The function block diagram of the demonstration platform is shown in Figure 6.

\section{Simulation Test Results}

\subsection{Advanced Simulator Design}

In [11], a number of simulators are designed by using typical random number 
generation functions in Matlab with Monte Carlo methods [19]. Considering that it is impractical to conduct actual trials for thousands of different scenarios, the normally distributed simulator, an evenly distributed simulator, and the behavior-based simulator are designed in accordance with [11]. Simulations are constructed by summing up individual appliance signatures from a database of 14 typical appliances and a total of 20 operating modes. Simulation results of three simulators are shown in Figure 7 - Figure 9.

\subsection{Identification Accuracy of Simulation}

Three designed simulators are used here to generate a series of OEs to test the identification accuracy of the proposed method under different signatures. The simulation results are shown in Figure 10. It can be seen that the proposed algorithm can achieve an accuracy of at least $90 \%$ under three simulators with different identification signatures.

\section{Laboratory Test Results}

\subsection{Identification accuracy}

A test bench platform is built in laboratory to verify the identification accuracy of the presented algorithm. Six different appliances including a fluorescent light (FL), a microwave oven (MO), an electric heater (EH), a water heater (WH), a water fountain (WF), and an induction cooker (IC) are connected under a main breaker. The voltage and current waveforms are recorded by a power recording device.

One hundred groups of voltage and current waveforms are recorded and processed in Matlab. Six different signal signatures such as current $(I)$, harmonic $(H)$, real and reactive power $(P Q)$, geometrical properties of the $V$-I curve and instantaneous power $(p)$ are employed separately to identify the appliances according to the procedure illustrated in Figure 1 . The identification accuracy of each appliance by each signature is shown in Table 1. The range of the accuracy is between $88 \%$ and $97 \%$ with a mean value of $92.8 \%$.

The identification accuracy of each appliance by multiple signatures is shown in Table 
2. The range of the accuracy is between $92 \%$ and $98 \%$ with a mean value of $94.3 \%$. It is known that the identification accuracy can be improved with multiple signatures.

In order to test the effect of the number of appliances on the identification accuracy, four additional appliances such as a fan (FAN), a washing machine (WM), a TV set (TV) and a vacuum cleaner (VC) are also involved in the following tests. The identification accuracy under conditions I, II, III and IV that involves four, six, eight and ten different appliances, respectively, is tested. The scheme is shown in Table 2. The average identification accuracy of several involved appliances under four conditions are shown in Table 3.

From Table 4, it can be easily observed that the presented appliance identification method has a very good accuracy of above $88 \%$ with ten different types of appliances.

\subsection{Laboratory tests}

Based on the experimental platform shown in Figure 4, the proposed algorithm was tested. Ten different appliance operation events (OEs) occur in sequence during the test as shown in Table 5. The symbol ' $\uparrow$ ' and ' $\downarrow$ ' means that an appliance is switched on and off, respectively.

The voltage at the point of common coupling (PCC) and six current signals are recorded by a data recording device. The six current signals include five currents from five appliances, respectively, and their total current at the PCC. The active power $(P)$ is selected as the signal signature for the appliance identification. Figure 11 illustrates the RMS curve of the active power and the OE number during the test.

The recorded data is processed by the presented algorithm in Matlab. The occurring time of each operation event (switching on or off) and the type of each appliance can be correctly detected with the selection of the signal signature of the active power, as shown in Figure 12.

The power consumption of each appliance can be calculated once the switching time is 
detected. The actual power consumption is calculated based on the voltage and branch current of each appliance. Table 5 shows the measurement accuracy of power consumption during the test duration time. It can be seen that the presented algorithm can achieve a satisfactory performance for monitoring home energy usage.

As opposed to the previous works in the literature based on intelligent systems [14]-[16] which are based on a large amount of training from operational data, the proposed work is based on the real-time measured data and does not require such effort work. It can reach roughly the same accuracy level as that in the literature. Note, the initial registration process is necessary for all NILM based approaches. Since this is a one-time initial process, it is a reasonably trivial effort.

\section{Conclusions}

This paper has presented a new appliance identification technique used in the NILM system for monitoring the operational status and decomposing power consumption information of each appliance in residential dwellings. The test result shows that the recognition is highly accurate (over $90 \%$ in general). The total electrical power consumption can be decomposed at each individual appliance level with errors less than $1 \%$. The output information is useful for scheduling loads and promoting the implementation of demand response programs. Further research work may include a longer time window (e.g., 1 day) of the presented technique and the identification in combination with several signal signatures.

\section{Acknowledgement}

The authors would like to acknowledge the support from the National Natural Science Foundation of China (51207088), Science and Technology Commission of Shanghai (14DZ1201602, 13DZ2251900), and the shared facility support provided by CURENT which is a US NSF/DOE Engineering Research Center funded under NSF award EEC-1041877. 


\section{References}

[1] K. Balasubramanian and A. Cellatoglu, "Improvements in Home Automation Strategies for Designing Apparatus for Efficient Smart Home,” IEEE Trans. Consumer Electron., vol. 54, no. 4, pp. 1681-1687, Nov. 2008.

[2] Jan Borstein, Karen Blackmore (March, 2008). In-Home Display Units: An Evolving Market, Part 1 (Report).

[3] Hart, G. W. "Prototype nonintrusive appliance load monitor," MIT Energy Laboratory Technical Report, and Electric Power Research Institute Technical Report, Sept. 1985.

[4] M. Zeifman, K. Roth, "Nonintrusive appliance load monitoring: Review and outlook," IEEE Trans. Consum. Electron, vol.57, no.1, pp.76-84, Feb. 2011.

[5] Pengfei Gao, Shunfu Lin, Wilsun Xu. A novel current sensor for home energy use monitoring, IEEE Transactions on Smart Grid. 21 Feb. 2014.

[6] Hsueh-Hsien Chang, Kun-Long Chen, et al, “A New Measurement Method for Power Signatures of Nonintrusive Demand Monitoring and Load Identification," IEEE Transactions on Industry Applications, , pp. $764-771,2012$.

[7] Jian Liang, S. Ng, G. Kendall, and J. Cheng. "Load Signature Study—Part I: Basic Concept, Structure, and Methodology," IEEE Transactions on Power Delivery, April 2010, vol. 25(2): $551-560$.

[8] K. D. Lee, "Electrical load information system based on non-intrusive power monitoring," Ph.D. thesis, Department of Mechanical Engineering, Massachusetts Institute of Technology, 2003.

[9] A. I. Cole and A. Albicki, "Nonintrusive identification of electrical loads in a three-phase environment base on harmonic content," Proc. of the 17th IEEE Instrumentation and Measurement Technology Conference (IMTC 2000), vol.1, May 1-4, 2000, pp.24 - 29.

[10] S B. Leeb, S R Shaw, et al, "Transient Event Detection in Spectral Envelope Estimates for Nonintrusive Load Monitoring," IEEE Transactions on Power Delivery, July 1995, Vol. 10, Iss. 3, pp. 1200-1211. 
[11] Q. Hu and F. Li, "Hardware Design of Smart Home Energy Management System with Dynamic Price Response," IEEE Transactions on Smart Grid, vol. 4, no. 4, pp. 1878-1887, Dec. 2013.

[12] Q. Hu, F. Li and C.-F. Chen, "A Smart Home Test Bed for Undergraduate Education to Bridge the Curriculum Gap Between Traditional Power Systems and Modernized Smart Grids," IEEE Transactions on Education, vol. 58, no. 1, pp-32-38, Feb. 2015.

[13] J. Liang, S. K. K. Ng, G. Kendall, J. W. M. Cheng, "Load Signature Study-Part II: Disaggregation Framework, Simulation, and Applications," IEEE Transactions on Power Delivery, vol. 25, pp. 561-569, 2010.

[14] Yu-Hsiu Lin, Men-Shen Tsai, "A Novel Signature Extraction Method for the development of nonintrusive load monitoring system based on BP-ANN," Computer Communication Control and Automation (3CA), 2010 International Symposium on, 2010, Page(s): 215 - 218.

[15] J. G. Roos, I .E. Lane, E. C. Lane, and G. P. Hanche, "Using neural networks for non-intrusive monitoring of industrial electrical loads," in Proc. IEEE Instrum. Meas. Technol. Conf., 1994, pp. $1115-1118$.

[16] D. Srinivasan, W. S. Ng, A. C. Liew, "Neural-Network-Based Signature Recognition for Harmonic Source Identification," IEEE Transactions on Power Delivery, vol. 21, pp. 398-405, 2006.

[17] AB Jambekar, DI Steinberg, "An implicit enumeration algorithm for the all integer programming problem," Computers \& Mathematics with Applications, 1978, 4(1):15-31.

[18] Page E. S. “Continuous Inspection Scheme,” Biometrika 41 (1/2): 100-115.

[19] R. Billinton and W. Li, "Reliability Assessment of Electric Power Systems Using Monte Carlo Methods," New York: Plenum, 1994. 

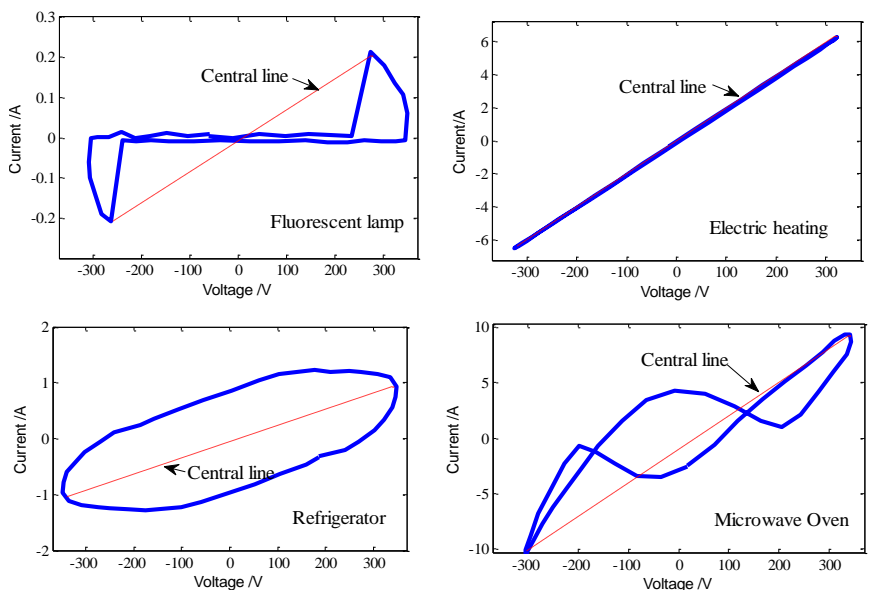

Figure 1: Processing procedure of the recording data.

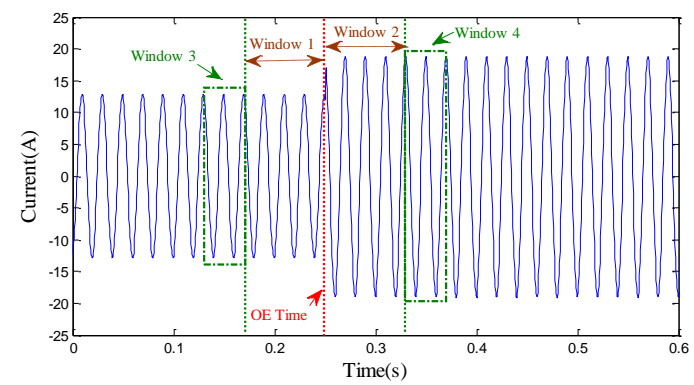

Figure 2: The principle diagram of the load characteristic database.

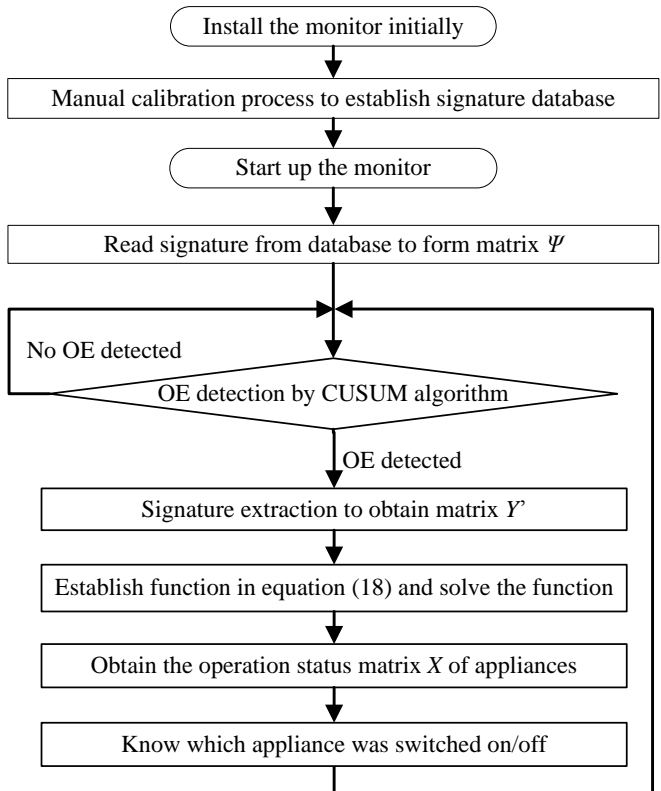

Figure 3: Processing procedure of the recording data. 


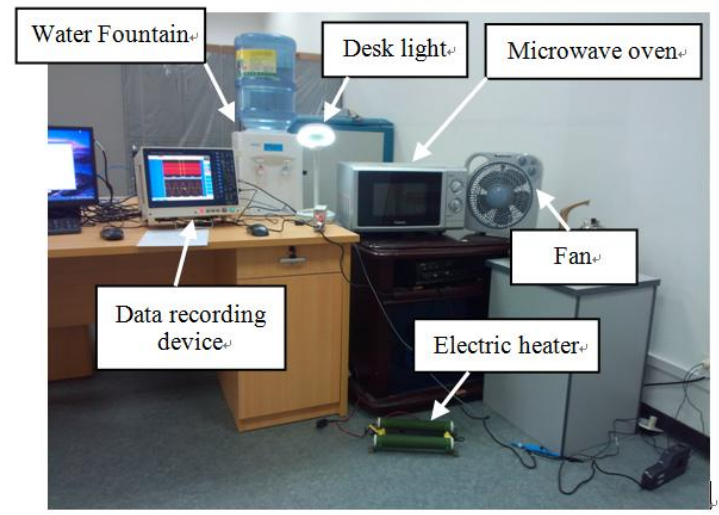

Figure 4: The experimental platform with five appliances.
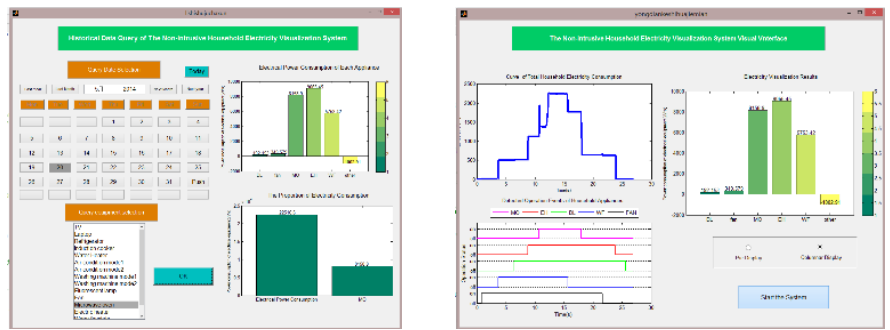

Figure 5: The Matlab GUI of the NILM demonstration platform.

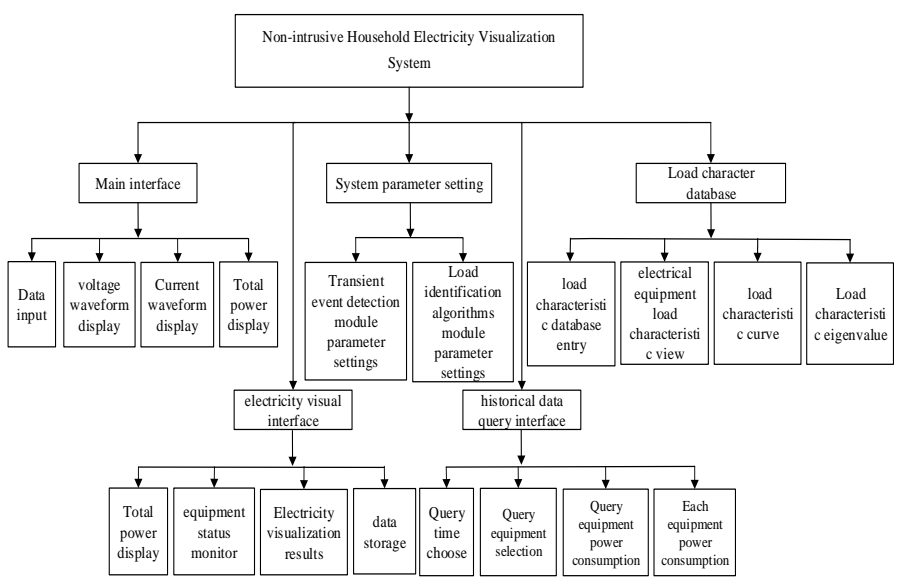

Figure 6: Overall functional block diagram of demonstration platform. 


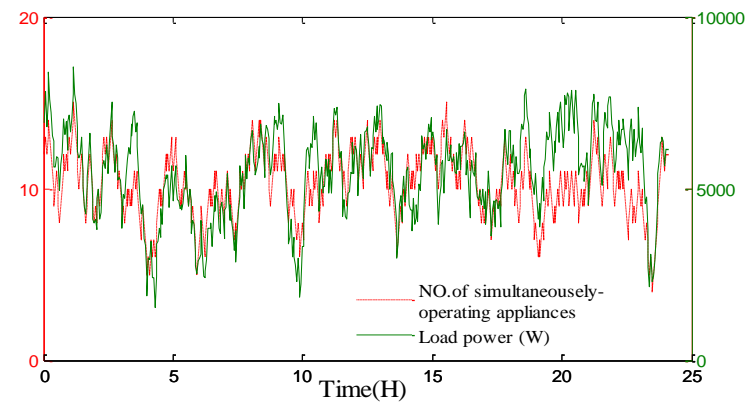

Figure 7: Simulation result of the normally distributed simulator.

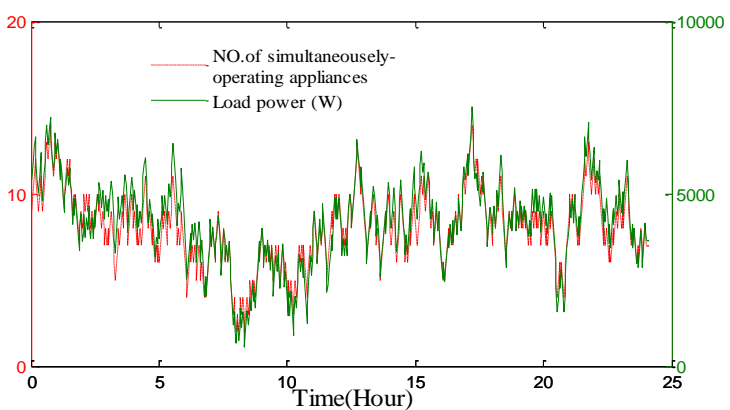

Figure 8: Simulation result of an evenly distributed simulator

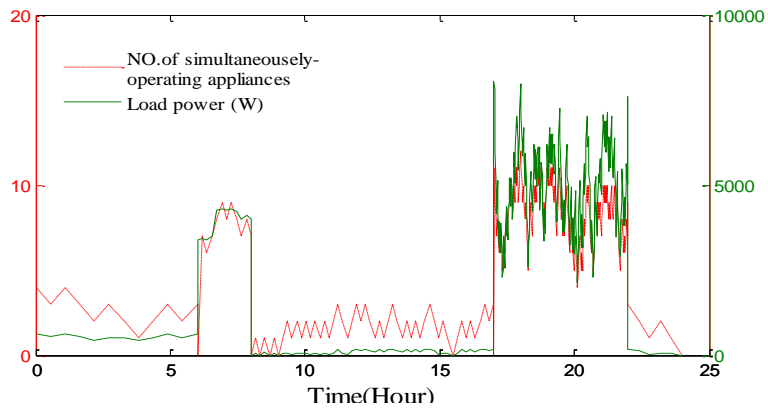

Figure 9: Simulation result of the behavioral-based simulator

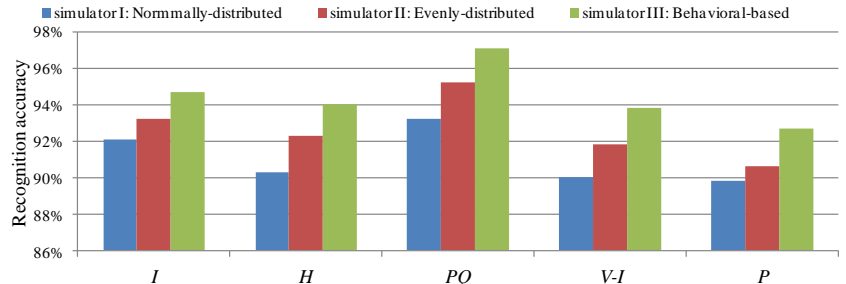

Figure 10: Accuracy under three simulators for different identification signatures. 


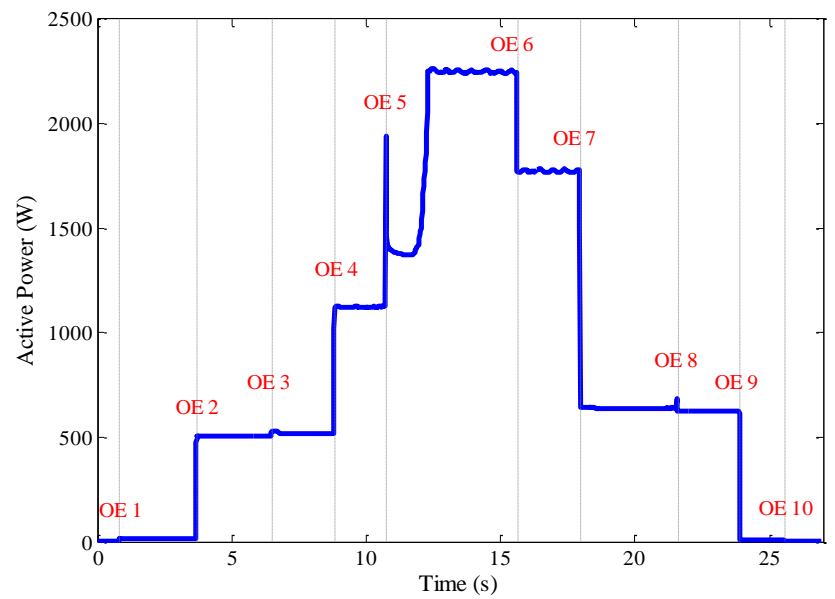

Figure 11: The RMS curve of the active power and OE number.

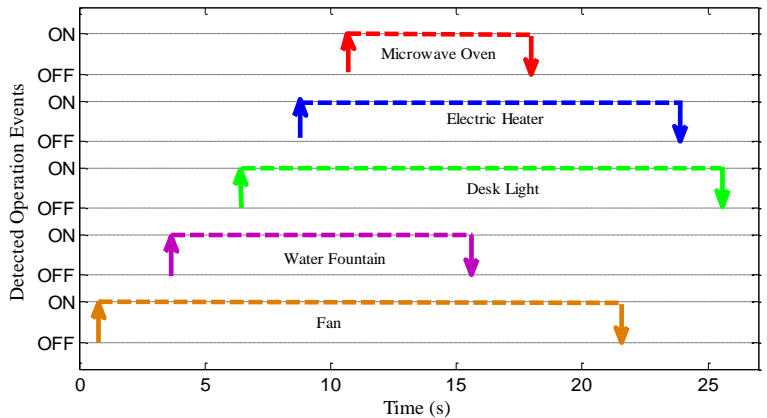

Figure 12: The detected operation events of five appliances. 
Table 1: Identification Accuracy of Each Appliance by Each Signature

\begin{tabular}{|c|c|c|c|c|c|}
\hline Appliances & Signatures & $\begin{array}{c}\text { Accuracy } \\
(\%)\end{array}$ & Appliances & Signatures & $\begin{array}{c}\text { Accuracy } \\
(\%)\end{array}$ \\
\hline \multirow{5}{*}{ FL } & $I$ & 92 & \multirow{5}{*}{ IC } & $I$ & 94 \\
\hline & $H$ & 94 & & $H$ & 93 \\
\hline & $P Q$ & 97 & & $P Q$ & 95 \\
\hline & $V-I$ & 93 & & $V-I$ & 91 \\
\hline & $p$ & 90 & & $p$ & 92 \\
\hline \multirow{5}{*}{ MO } & $I$ & 92 & \multirow{5}{*}{ WF } & $I$ & 92 \\
\hline & $H$ & 89 & & $H$ & 94 \\
\hline & $P Q$ & 93 & & $P Q$ & 96 \\
\hline & $V-I$ & 88 & & $V-I$ & 93 \\
\hline & $p$ & 91 & & $p$ & 90 \\
\hline \multirow{5}{*}{ WH } & $I$ & 94 & \multirow{5}{*}{$\mathrm{EH}$} & $I$ & 94 \\
\hline & $H$ & 93 & & $H$ & 91 \\
\hline & $P Q$ & 96 & & $P Q$ & 96 \\
\hline & $V-I$ & 94 & & $V-I$ & 92 \\
\hline & $p$ & 92 & & $p$ & 92 \\
\hline
\end{tabular}

Table 2: Recognition Accuracy of Each Appliance by Multiple Signatures

\begin{tabular}{|c|c|c|c|c|c|}
\hline Appliances & Signatures & $\begin{array}{c}\text { Accuracy } \\
(\%)\end{array}$ & Appliances & Signatures & $\begin{array}{c}\text { Accuracy } \\
(\%)\end{array}$ \\
\hline \multirow{4}{*}{ FL } & $I+H$ & 92 & \multirow{4}{*}{ IC } & $I+H$ & 94 \\
\hline & $P Q+H$ & 94 & & $P Q+H$ & 94 \\
\hline & $P Q+V-I$ & 98 & & $P Q+V-I$ & 95 \\
\hline & $P Q+H+V-I$ & 98 & & $P Q+H+V-I$ & 94 \\
\hline \multirow{4}{*}{ MO } & $I+H$ & 90 & \multirow{4}{*}{ EH } & $I+H$ & 92 \\
\hline & $P Q+H$ & 92 & & $P Q+H$ & 95 \\
\hline & $P Q+V-I$ & 93 & & $P Q+V-I$ & 96 \\
\hline & $P Q+H+V-I$ & 96 & & $P Q+H+V-I$ & 95 \\
\hline
\end{tabular}

Table 3: Four Test Conditions with Different Appliances

\begin{tabular}{c|c}
\hline Condition & Appliances \\
\hline I & WF+WH+FL+EH \\
\hline II & WF+MO+IC+FL+EH \\
\hline III & FL+IC+WF+WH+MO+EH+TC+VC \\
\hline IV & FL+IC+WF+WH+MO+EH+VC+TV+WM+FAN \\
\hline
\end{tabular}

Table 4: Identification Accuracy of Load Identification Algorithms of Different Types of Equipment

\begin{tabular}{|c|c|c|c|c|c|}
\hline Condition & Signatures & $\begin{array}{c}\text { Accuracy } \\
(\%)\end{array}$ & Condition & Signatures & $\begin{array}{c}\text { Accuracy } \\
(\%)\end{array}$ \\
\hline \multirow{5}{*}{ I } & $I$ & 96 & \multirow{5}{*}{ III } & $I$ & 92 \\
\hline & $H$ & 96 & & $H$ & 91 \\
\hline & $P Q$ & 98 & & $P Q$ & 95 \\
\hline & $V-I$ & 97 & & $V-I$ & 92 \\
\hline & $p$ & 95 & & $p$ & 90 \\
\hline \multirow{5}{*}{ II } & $I$ & 94 & \multirow{5}{*}{ IV } & $I$ & 90 \\
\hline & $H$ & 93 & & $H$ & 88 \\
\hline & $P Q$ & 96 & & $P Q$ & 93 \\
\hline & $V-I$ & 93 & & $V-I$ & 90 \\
\hline & $p$ & 92 & & $p$ & 89 \\
\hline
\end{tabular}


Table 5: Ten Operation Events that Occurred During the Test

\begin{tabular}{c|c|c|c|c|c}
\hline OE No. & 1 & 2 & 3 & 4 & 5 \\
\hline $\mathrm{OE}$ & FAN $\uparrow$ & WF $\uparrow$ & $\mathrm{DL} \uparrow$ & $\mathrm{EH} \uparrow$ & $\mathrm{MO} \uparrow$ \\
\hline $\mathrm{OE}$ No. & 6 & 7 & 8 & 9 & 10 \\
\hline $\mathrm{OE}$ & $\mathrm{WF} \downarrow$ & $\mathrm{MO} \downarrow$ & $\mathrm{FAN} \downarrow$ & $\mathrm{EH} \downarrow$ & $\mathrm{DL} \downarrow$ \\
\hline
\end{tabular}

Table 6: Power Consumption Measurement Accuracy

\begin{tabular}{c|c|c|c}
\hline \multirow{2}{*}{ Appliance } & $\begin{array}{c}\text { Actual power consumption } \\
(\mathrm{Ws})\end{array}$ & $\begin{array}{c}\text { Measured power consumption } \\
(\mathrm{Ws})\end{array}$ & $\begin{array}{c}\text { Error } \\
(\%)\end{array}$ \\
\hline FAN & 347.0 & 345.7 & -0.37 \\
\hline DL & 190.4 & 190.0 & -0.21 \\
\hline WF & 5717.5 & 5664.0 & -0.94 \\
\hline EH & 9063.9 & 9000.0 & -0.70 \\
\hline WO & 8108.4 & 8175.2 & 0.82 \\
\hline Total & 23425 & 23374.9 & -0.21 \\
\hline
\end{tabular}

